\title{
Recurrent mutations at C-reactive protein gene promoter SNP position -286 in human cancers
}

Cell Research (2014) 24:505-508. doi:10.1038/cr.2014.7; published online 14 January 2014

\section{Dear Editor,}

Genetic alteration and inflammation underlie the development of cancer [1]. C-reactive protein (CRP) is the most widely used nonspecific marker of inflammation [2], whose serum level has been shown to be associated with the risk and prognosis of several types of cancer [3]. Accumulating evidence supports a role of CRP as a pattern recognition receptor in the innate immunity and inflammation [4]; however, its exact function remains to be defined because mouse is not an appropriate animal model for CRP [5]. Moreover, as a prototypical acute phase reactant, the 2-3-order fluctuation in the serum level of CRP has raised the concern that this molecule is not likely a fine modulator of inflammation [2]. These make it difficult to dissect the contribution of CRP in diseases featured by chronic inflammation, including atherosclerosis [2] and cancer [3]. What makes things even more complicated is the fact that large-scale genetic epidemiologic studies do not support a causal association of CRP with these diseases $[6,7]$. Therefore, it remains elusive whether CRP is a passive marker or plays a direct role in tumorigenesis.

Recent studies have demonstrated that in addition to hepatocytes, CRP can also be produced by extra-hepatic cells [2]. In searching for mechanism(s) involved in the regulation of $C R P$ expression in human cancer cells, we incidentally found that the T-cell leukemia cell line Jurkat and the hepatoma cell line Hep3B appeared to possess the CA genotype, instead of the predominant $\mathrm{CC}$ genotype, at the position of 286 bp upstream of the TSS of $C R P$ (Figure 1A). Further screening identified only 6 cases of $-286 \mathrm{CC}$ genotype of additional 13 cancer cell lines. The $-286 \mathrm{C}>\mathrm{A} / \mathrm{T}$ substitution is a known SNP of $C R P$ (rs3091244), which is associated with elevated plasma concentrations of CRP [6]. However, it is less likely that the cancer cell lines with the $-286 \mathrm{CA} / \mathrm{CT}$ genotype all happened to be derived from individuals carrying the minor alleles. Alternatively, the $-286 \mathrm{C}>\mathrm{A} / \mathrm{T}$ transition may arise as a mutation during cancer development in the original hosts or during prolonged passage of the established cell lines.

With the above hint, we sequenced the promoter of $C R P$ in 453 matched tumor/normal sample pairs. Overall, 109 tumors (24\%) harbor -286 somatic mutations, the majority of which are $-286 \mathrm{C}>\mathrm{A}$ (Figure $1 \mathrm{~B}$ and Supplementary information, Tables S1 and S2). The -286 mutations were detected in 10 of 12 cancer types with the strongest prevalence $(53.3 \%)$ for colon cancer (Figure 1C). The fractions of the mutated -286 allele are high in tumors (the average allelic fraction is $0.497 ; 95 \% \mathrm{CI}$ : 0.477 to 0.517 ) and these mutations exhibit comparable mutational rates in frozen and formalin-fixed sample pairs. No additional recurrent mutations were identified within the near $700 \mathrm{bp}$ sequenced range of $C R P$ promoter $(-601 \sim+76)$, which includes 14 other known CRP SNP sites. To exclude the possible misidentification of the -286 germline SNP as a somatic mutation, regular PCR sequencing results of 73 sample pairs were independently validated by TA clone sequencing (Supplementary information, Tables S2 and S3). Moreover, we genotyped 20 randomly selected SNP sites of 141 tumor/normal sample pairs, in which $123(87.23 \%)$ were completely matched (Supplementary information, Table S4). This further demonstrate that the identified $C R P-286$ mutations were not due to mismatch of sample pairs or technical artifacts. Therefore, we conclude that the promoter of $C R P$ is specifically mutated at position -286 during cancer development.

The mRNA levels of $C R P$ in tumors were much higher than that in matched controls (Figure 1D). Interestingly, the relative induction of $C R P$ expression tends to be more pronounced in tumors with $-286 \mathrm{C}>\mathrm{A}$ somatic mutation (Figure 1E), suggesting that this mutation enhances $C R P$ transcription. Indeed, $C R P$ promoter construct carrying the -286 A allele shows higher luciferase reporter activity than the wild-type construct [8].

Promoter $\mathrm{CpG}$ methylation is an essential epigenetic mechanism in gene silencing. There are only $5 \mathrm{CpG}$ dinucleotides, including the one containing -286 cytosine, within the range of $700 \mathrm{bp}$ upstream of the TSS of $C R P$ gene. Rabbit exhibits similar $C R P$ expression pattern 
A $\quad$-286C>A mutation

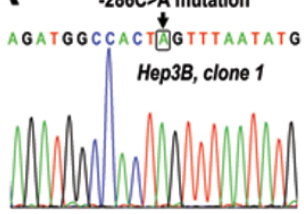

AGATGGCCACTCGTTTAATATG

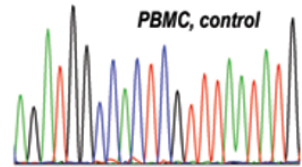

C

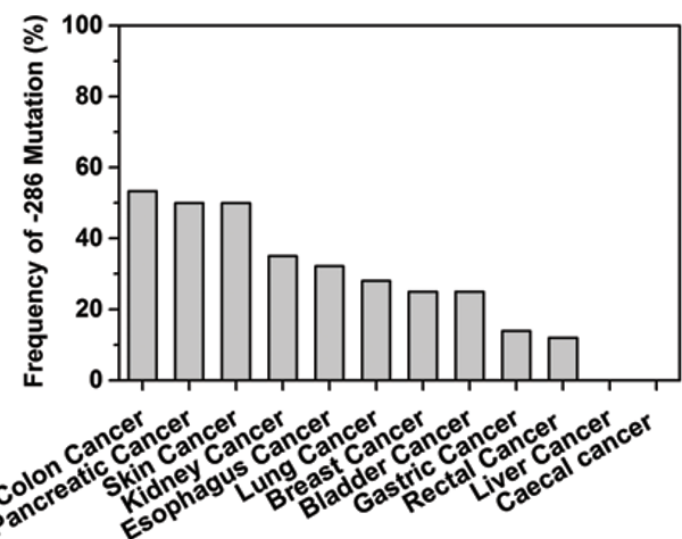

B

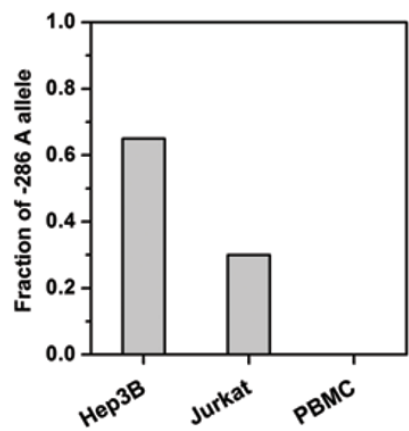

\begin{tabular}{|c|c|c|c|}
\hline Cancer Type & $\begin{array}{c}\text { Number of } \\
\text { Tumor/Normal } \\
\text { Sample Pairs }\end{array}$ & $\begin{array}{c}\text { Number of } \mathbf{- 2 8 6} \\
\text { Somatic Mutation in } \\
\text { Tumors (\%) }\end{array}$ & $\begin{array}{c}\text { Number of }-286 C>A \\
\text { Somatic Mutation in } \\
\text { Tumors }(\%)\end{array}$ \\
\hline \multicolumn{4}{|l|}{ Bladder Cancer } \\
\hline Formalin-fixed & 4 & $1(25.0)$ & $1(25.0)$ \\
\hline \multicolumn{4}{|l|}{ Breast Cancer } \\
\hline Formalin-fixed & 8 & $2(25.0)$ & $1(12.5)$ \\
\hline \multicolumn{4}{|l|}{ Colon Cancer } \\
\hline Formalin-fixed & 13 & $8(61.5)$ & $8(61.5)$ \\
\hline Frozen & 32 & $16(50.0)$ & $14(43.8)$ \\
\hline \multicolumn{4}{|l|}{ Esophagus Cancer } \\
\hline Formalin-fixed & 9 & $3(33.3)$ & $2(22.2)$ \\
\hline Frozen & 75 & $24(32.0)$ & $13(17.3)$ \\
\hline \multicolumn{4}{|l|}{ Gastric Cancer } \\
\hline Formalin-fixed & 51 & 7 (13.7) & $6(11.8)$ \\
\hline Frozen & 100 & $14(14.0)$ & $10(10.0)$ \\
\hline \multicolumn{4}{|l|}{ Kidney Cancer } \\
\hline Formalin-fixed & 20 & $7(35.0)$ & $2(10.0)$ \\
\hline \multicolumn{4}{|l|}{ Lung Cancer } \\
\hline Formalin-fixed & 11 & $2(18.2)$ & $1(9.1)$ \\
\hline Frozen & 39 & $12(30.8)$ & $3(7.7)$ \\
\hline \multicolumn{4}{|l|}{ Pancreatic Cancer } \\
\hline Formalin-fixed & 4 & $2(50.0)$ & $1(25.0)$ \\
\hline \multicolumn{4}{|l|}{ Rectal Cancer } \\
\hline Formalin-fixed & 4 & $1(25.0)$ & $0(0.0)$ \\
\hline Frozen & 71 & $8(11.3)$ & $3(4.2)$ \\
\hline \multicolumn{4}{|l|}{ Skin Cancer } \\
\hline Formalin-fixed & 4 & $2(50.0)$ & $2(50.0)$ \\
\hline
\end{tabular}

D

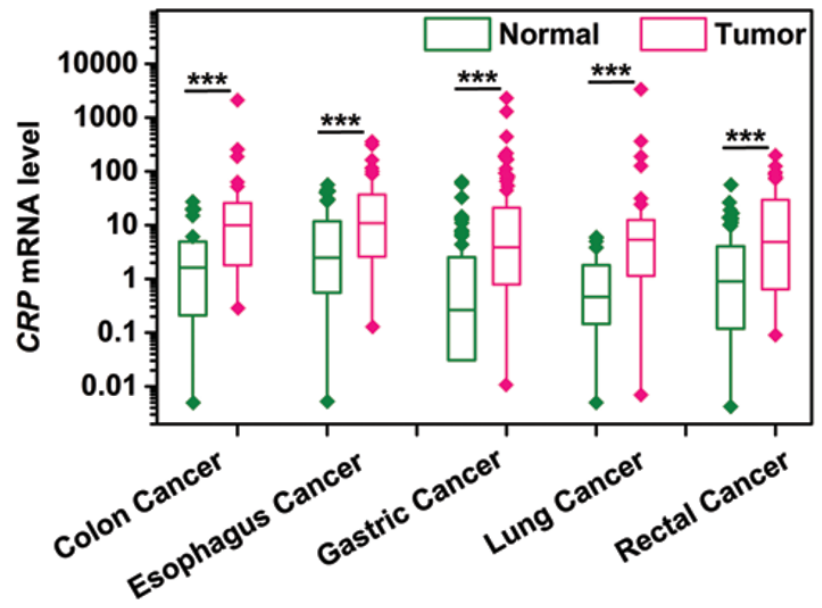

E

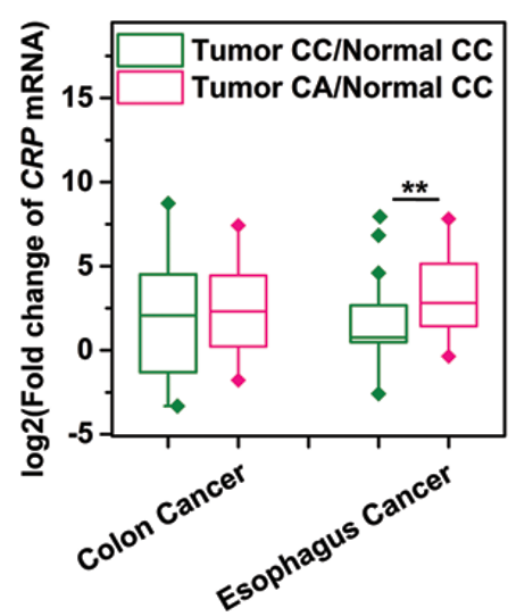

Figure 1 Recurrent -286 promoter mutation of CRP. (A) High fractions of $-286 \mathrm{C}>\mathrm{A}$ substitutions were found in Hep3B and Jurkat cell lines but not in PBMC control cells. Clone sequencing results (left) and -286 A allelic frequencies (right) are shown. (B) CRP promoter sequences in 453 tumor/normal sample pairs were determined. 67 tumors acquired $-286 \mathrm{C}>\mathrm{A}$ mutation with additional 42 tumors exhibiting other types of -286 mutation. Two cancer types (caecal cancer, $n=3$; liver cancer, $n=5$ ) without detected -286 mutation are not listed. (C) The frequencies of -286 mutation in different cancer types are shown. (D) CRP mRNA expressions in frozen tumors and matched normal controls of colon $(n=32)$, esophagus $(n=74)$, gastric $(n=96)$, lung $(n=38)$ and rectal cancers $(n=67)$ were determined by q-PCR. CRP mRNA levels are significantly higher in tumors $\left.{ }^{\star \star \star} P<0.001\right)$. The statistical analysis was performed by the two-tailed paired sample Wilcoxon Signed Rank test. The results of other cancer types are not shown due to insufficient sample sizes. (E) The relative inductions of CRP expression in tumors with or without $-286 \mathrm{C}>\mathrm{A}$ somatic mutation over matched controls were calculated. Results are shown for colon and esophagus cancers, which have sufficient sample sizes and exhibit high frequencies of $-286 \mathrm{C}>\mathrm{A}$ mutation. Tumors with $-286 \mathrm{C}>\mathrm{A}$ mutation tend to show stronger induction of CRP expression, although statistical significance is reached only for esophagus cancer $\left({ }^{* *} P<0.01\right)$. The statistical analysis was performed by the two-tailed Mann-Whitney test. 
to human and preserves the $\mathrm{CpG}$ site corresponding to human -286 CpG (Supplementary information, Figure $\mathrm{S} 1 \mathrm{~A})$. Rabbit $C R P$ was primarily expressed by the liver (Supplementary information, Figure S1B) and all the $\mathrm{CpG}$ sites in the CRP promoter were largely unmethylated in hepatocytes (Supplementary information, Figure S1C). Moreover, the muscle tissue of rabbit exhibited appreciable expression only at the $-308 \mathrm{CpG}$, which corresponds to the $-286 \mathrm{CpG}$ in humans. The other tissues of rabbit, by contrast, showed essentially no CRP expression and high levels of methylation at all promoter $\mathrm{CpG}$ sites. These results suggest that $C R P$ expression may be at least partly determined by the status of promoter methylation, in particular the $-286 \mathrm{CpG}$, under normal and less stressed/pre-malignant conditions. Further experiments showed apparently reduced methylation levels of $C R P$ promoter in Hep3B cells upon stimulation with IL-6 and IL-1, which significantly increased the expression of CRP (Supplementary information, Figure S1D and S1E), implying that promoter demethylation also contributes to induced expression of $C R P$ in cancer cells. Together, the above results indicate that the disruption of the conserved promoter $\mathrm{CpG}$ methylation motif may be an important mechanism for the -286 somatic mutations to enhance the levels of $C R P$ in both early and late stages of tumorigenesis.

The high prevalence of $-286 \mathrm{C}>\mathrm{A}$ mutation in the absence of recurrent mutations at other positions in the promoter of $C R P$ is consistent with the interpretation that this mutation is intimately involved in tumorigenesis. Indeed, recurrent promoter mutations in TERT have recently been identified as driver events in melanoma [ 9 , 10]. These constitute the first examples that regulatory regions of key genes, in addition to coding sequences, can also be targeted for somatic mutations to regulate cancer development. In this regard, it is intriguing that the dysregulation of CRP, a secretory effector of inflammation but not an intracellular transcription factor or kinase, is selected, presumably to confer host cells sufficient growth advantage to survive and expand in the clone competition accompanying tumorigenesis. This argues that CRP may be a core component deeply embedded in the regulatory network of inflammation, and suggests that regulatory sequences of other inflammatory effector genes could also be targeted in tumor cells.

The recurrence of the $-286 \mathrm{C}>\mathrm{A}$ promoter mutation in several cancer types suggests that CRP may be involved in general mechanisms favoring tumorigenesis. Although the acute phase reactant nature of human CRP appears to be incompatible with its function in regulating sophisticated cellular processes, continuous efforts of our and other groups have revealed that regulated conformation changes in CRP underlies its action as a fine modulator of inflammatory responses [2]. As triggers of these conformational changes are enriched in inflammatory lesions [2], the stressful tumor microenvironment provides an ideal setting for the localized expression of the full repertoire of CRP activities. As such, the local abundance but not the serum level of CRP is more relevant to disease status. Consequently, CRP, depending on its conformation, may regulate cancer development by preventing cancer cell apoptosis, by facilitating invasiveness and by promoting angiogenesis $[2,11,12]$. It is also likely that CRP may help cancer cells to escape or resist the attack from the immune system through induction of inflammatory cytokine release, and inhibition of destructive complement activation [2].

The $-286 \mathrm{C}>\mathrm{A}$ SNP has been reported to be associated with an elevated CRP concentration but not an increased risk of cancer [6]. Nonetheless, as stated by the authors [6], combining a heterogeneous group of cancers into a single outcome limits applicability of their results to specific cancer types. Indeed, the -286 CA genotype appears to be enriched in patients with rectal cancer $(57.8 \%$; odds ratio $1.78,95 \% \mathrm{CI}: 1.02$ to 3.09 ) but depleted in patients with colon cancer $(4.4 \%$; odds ratio $0.16,95 \%$ CI: 0.03 to 0.66 ) (Supplementary information, Table S2) when compared with that of 274 sequenced healthy controls $(23 \%)$. The enrichment of this SNP in specific cancer type, and the high recurrence and pervasiveness of -286 mutations in tumors together argue that the actions of CRP are primarily dedicated by specific cell types and the tumor-prone microenvironment.

Collectively, we demonstrate for the first time that a germline SNP in the non-coding region of a gene can be acquired as a somatic mutation with high recurrence and specificity during the development of cancer. This raises the possibility that known SNPs, particularly those of inflammatory effector genes, may represent a rich set for the discovery of cancer-contributing mutations in regulatory non-coding regions. Moreover, the $-286 \mathrm{C}>\mathrm{A}$ promoter mutation of $C R P$ exemplifies an elegant mechanism by which a targeted genetic alteration can be coupled to the epigenetic dysregulation of gene expression in tumors. Further investigations are warranted to resolve the overlooked activities of CRP in cancer development and validate the potential usage of the $-286 \mathrm{C}>\mathrm{A}$ SNP for cancer risk screening. Given the highly induced expression of CRP in tumors, topical modulation of activities of CRP may be a potential option for cancer therapy.

Experimental materials and methods are depicted in the Supplementary information, Data S1. 


\section{Acknowledgments}

This work was supported by grants from the MOST (2011CB910500) and NSFC (30930024, 31222015, 31270813, 31170696) and MOE (PCSIRT: IRT1137, 121108).

\section{Ming-Yu Wang ${ }^{1, *}$, Hai-Hong Zhou ${ }^{1, *}$, Shi-Chao Zhang ${ }^{1, *}$,} Feng Hui ${ }^{1, *}$, Wei $\mathrm{Zhu}^{2}$, Hai-Xiang $\mathrm{Su}^{3}$, Hong-Yun Guo ${ }^{3}$, Xing-Wen $\mathrm{Li}^{3}$, Shang-Rong $\mathrm{Ji}^{1}$, Yi Wu ${ }^{1,4}$

${ }^{1}$ MOE Key Laboratory of Cell Activities and Stress Adaptations, School of Life Sciences, Lanzhou University, Lanzhou, Gansu 730000, China; ${ }^{2}$ the Second Hospital, Lanzhou University, Lanzhou, Gansu 730000, China; ${ }^{3}$ The Gansu Cancer Hospital, Lanzhou 730050, China: ${ }^{4}$ Key Laboratory of Preclinical Study for New Drugs of Gansu Province, Lanzhou University, Lanzhou, Gansu 730000, China

*These four authors contributed equally to this work.

Correspondence: $\mathrm{Yi} \mathrm{Wu}^{\mathrm{a}}$, Shang-Rong $\mathrm{Ji}^{\mathrm{b}}$

${ }^{\mathrm{a}}$ Tel/Fax: +86-931-8914102

E-mail: wuy@1zu.edu.cn
${ }^{\mathrm{b}} \mathrm{Tel} / \mathrm{Fax}:+86-931-8914176$

E-mail: jsr@1zu.edu.cn

\section{References}

1 Hanahan D, Weinberg RA. Cell 2011; 144:646-674.

2 Ma X, Ji SR, Wu Y. Chinese Sci Bull 2013; 58:1642-1649.

3 Allin KH, Nordestgaard BG. Crit Rev Clin Lab Sci 2011; 48:155-170.

4 Bottazzi B, Doni A, Garlanda C, Mantovani A. Annu Rev Immunol 2010; 28:157-183.

5 Reifenberg K, Lehr HA, Baskal D, et al. Arterioscler Thromb Vasc Biol 2005; 25:1641-1646.

6 Allin KH, Nordestgaard BG, Zacho J, et al. J Natl Cancer Inst 2010; 102:202-206

7 Zacho J, Tybjaerg-Hansen A, Jensen JS, et al. N Engl J Med 2008; 359:1897-1908.

8 Kim HA, Chun HY, Kim SH, et al. J Rheumatol 2009; 36:2238-2243.

9 Huang FW, Hodis E, Xu MJ, et al. Science 2013; 339:957-959.

10 Horn S, Figl A, Rachakonda PS, et al. Science 2013; 339:959-961.

11 Yang J, Wezeman M, Zhang X, et al. Cancer Cell 2007; 12:252-265.

12 Kim ES, Cha Y, Ham M, et al. Oncogene 2013 Aug 19. doi: 10.1038/ onc. 2013.319

(Supplementary information is linked to the online version of the paper on the Cell Research website.) 